\title{
Optical properties of InSb layers confined by InP
}

\author{
T. Utzmeier, G. Armelles, P. A. Postigo, and F. Briones \\ Instituto de Microelectrónica de Madrid, CNM, CSIC, Isaac Newton 8, 28760 Tres Cantos, Spain
}

(Received 27 January 1997; revised manuscript received 14 April 1997)

\begin{abstract}
The photoluminescence properties of InSb submonolayers and dots are presented. When the amount of InSb deposit on InP is less than $1 \mathrm{ML}$ the growth mode remains two dimensional, while for greater amounts three-dimensional quantum dots are formed. Within the two-dimensional growth mode range the energy of the InSb-related transition decreases as we increase the amount of InSb deposited within the limits of said range. No InSb absorption features have been detected. The observed photoluminescence is interpreted as a recombination of electrons in the InP layers with holes in the InSb layers. We estimate a valence-band offset of 1.525 $\mathrm{eV}$, similar to the predictions of the solid-model theory $(1.600 \mathrm{eV})$. In the samples with InSb dots an InSb related transition has been observed, which is attributed to the wetting layer. [S0163-1829(97)04032-0]
\end{abstract}

InSb bulk material has been intensively studied in the past because of its promising properties like the extremely high electron bulk mobility of $92000 \mathrm{~cm}^{2} / \mathrm{V} \mathrm{s}$ (at $77 \mathrm{~K}$ ) (Ref. 1) and low band gap of $0.17 \mathrm{eV}($ Ref. 2) at $300 \mathrm{~K}$. Despite this interest very little is known about thin InSb heterostructures. In this paper we present an optical study of InSb/InP single heterostructures. Due to the lattice mismatch between InSb and InP $(10.4 \%)$ the growth mode of InSb on InP quickly transforms from a two-dimensional layer-by-layer growth to a three-dimensional island growth. This transition occurs for depositions of InSb exceeding 1.2 ML. ${ }^{3}$ We analyze the optical properties of samples having an amount of InSb lower than $1 \mathrm{ML}$, where a clear two-dimensional growth mode is observed, and samples having an amount of $\mathrm{InSb}$ greater than $1 \mathrm{ML}$, where self-assembled InSb quantum dots (QD's) are obtained.

The samples were grown by molecular-beam epitaxy. During the growth the In element was continuously supplied, whereas the group-V element was pulsed. The amount of group- $\mathrm{V}$ element deposited on the sample was controlled by the reflection difference technique, ${ }^{4}$ which measures the coverage of surface In dimers. The submonolayers and $1 \mathrm{ML}$ samples were grown as follows: After the growth of a InP buffer layer at $400{ }^{\circ} \mathrm{C}$ the substrate temperature was reduced to $300{ }^{\circ} \mathrm{C}$, then $20 \mathrm{ML}$ of InP were grown followed by the $\mathrm{InSb}$ deposition and continued by the growth of another 20 ML of InP. After that the substrate temperature was risen up again to grown $180 \mathrm{ML}$ of InP. The growth temperature was reduced during the deposition of the $\mathrm{InSb}$, to reduce surface migration and assure a two-dimensional growth mode. In order to better control the InSb thickness, the InSb was deposited at a growth rate of $0.25 \mathrm{ML} / \mathrm{s}$, whereas the InP was growth at $0.5 \mathrm{ML} / \mathrm{s}$. For the growth of the samples with InSb amounts greater than $1 \mathrm{ML}$ the temperature reduction of the substrate was not carried out since island growth was pretended. After the formation of the InSb islands, clearly observed by reflection high-energy electron diffraction, an annealing at $440{ }^{\circ} \mathrm{C}$ under $\mathrm{Sb}_{2}$ flux was performed to enhance the dot uniformity. The InSb dots were also covered by a 200-m InP cap-layer grown at $400{ }^{\circ} \mathrm{C}$.

In Fig. 1 we present the photoluminescence (PL) spectra of some samples studied here. Those spectra were obtained at $12 \mathrm{~K}$ using a liquid-nitrogen cooled Ge detector and exciting with the 488-nm line of an $\mathrm{Ar}^{+}$at $24 \mathrm{~W} / \mathrm{cm}^{2}$. Together with the InP-related transitions another peak, related to the InSb deposition, is observed. As expected, when we increase the amount of InSb from 0.3 to $1 \mathrm{ML}$ the InSbrelated peak decreases in energy. Surprisingly, for $\mathrm{InSb}$ depositions greater than $1 \mathrm{ML}$ (three-dimensional growth mode), the InSb-related transition is located at an energy higher than that of the $1 \mathrm{ML}$ sample. For example, in Fig. 1 we present the spectrum of a two monolayers sample. The energy position of this transition does not depend strongly on the amount of InSb. No electro- or piezoreflectance features related to the InSb deposition have been detected.

The theoretical predictions of the valence-band offset between InSb and InP vary between 0.750 (Ref. 5) and 1.18 eV. ${ }^{6}$ These values correspond to systems where no strain has been taken into account. The strain present in the layers modifies the said alignment. In particular, for InSb lattice matched to InP due to the difference between the hydrostatic deformation potentials of the conduction-band minimum and valence-band maximum, ${ }^{7}$ both theoretical calculations suggest a type-II band alignment between InSb and InP. For example, in Fig. 2 we present the band alignment between InSb and InP suggested by the solid-model theory. ${ }^{6}$ Figure

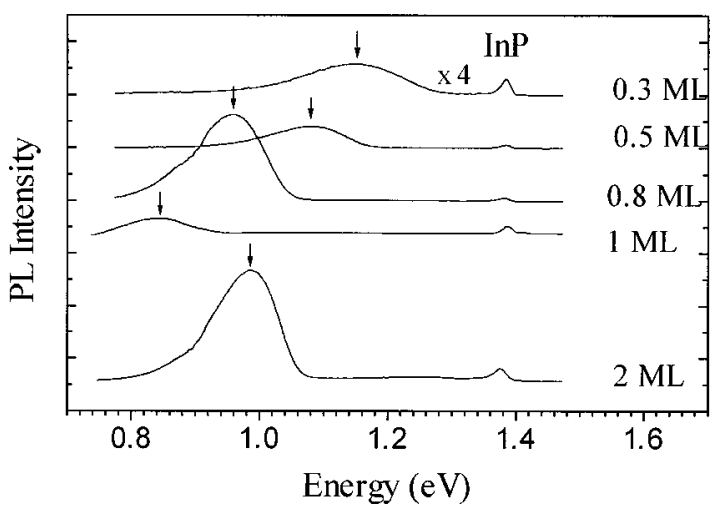

FIG. 1. PL spectra of samples with various InSb layer thickness, ranging from 0.3 to $2 \mathrm{ML}$. From 0.3 to $1 \mathrm{ML}$ the InSb layer is two-dimensional, while the 2-ML sample presented QD's. The arrows indicate the InSb-related emission. 
(a)

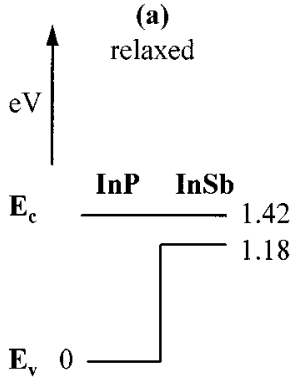

(b)

pseudomorphic

(c)

hydrostatic compression

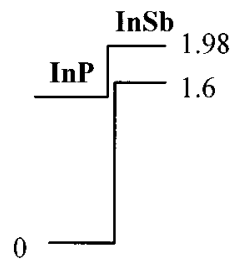

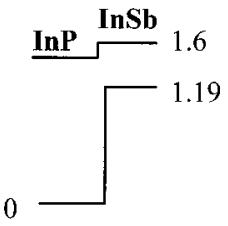

FIG. 2. Band alignments between InSb and InP for different strain states: (a) fully relaxed, (b) InSb coherently grown on InP, and (c) InSb with a hydrostatic compression of $\varepsilon \approx 1 \%$. The values of the different deformation potentials are taken from Ref. 6.

2(a) corresponds to a situation where both InP and InSb are unstrained and Fig. 2(b) corresponds to a situation where the InSb is lattice matched to InP. The conduction-band minimum of $\mathrm{InSb}$ is shifted due to the hydrostatic component of the strain, whereas the valence-band maximum is shifted mainly due to the shear component of the strain. For clarity the light-hole band is not shown. The situation of Fig. 2(b) corresponds to the ML sample, we associate the observed PL transition with the recombination of electrons in the InP confining material with heavy holes localized in the InSb regions. Due to the spatial separation of the electrons and holes the intensity of absorption-related features is expected to be small, in agreement with our piezo- and electroreflectance results. Additionally, we have observed a blueshift in the PL spectra of $27 \mathrm{meV}$ when the excitation power was increased by two orders of magnitude. Such a blueshift is typical for type-II lineup heterostructures. ${ }^{8}$ Using this theoretical estimation of the valence-band offset (VBO) between InSb and InP we can calculate the energy position of the transition for a 1-ML quantum well (QW) of InSb in InP using the masses of the heavy holes reported in the literature ${ }^{2}$ which amounts to $0.834 \mathrm{eV}$, near to the experimental value of $0.87 \mathrm{eV}$. A better agreement can be obtained using a VBO of $1525 \mathrm{meV}$, which is our estimation of the VBO between InSb lattice matched to InP. Just for completeness, in Fig. 3 we present the theoretical values of the transition for the submonolayers samples. In this calculation we have considered that the submonolayer can be viewed as a monolayer of an alloy with the same equivalent composition. As can be observed, when the amount of InSb is decreased the disagreement between the theoretical estimation and the energy of the transition increases. The discrepancy between theory and experiment can be explained assuming that the InSb nucleated in form of two-dimensional islands on the InP surface and is not distributed homogeneously. The existence of these islands is supported by the polarization behavior of the InSb-related emission, shown in Fig. 4. In that figure we present the difference between the intensity of the InSb emission for light polarized along [110] and [1 $\overline{10}$ ]. A maximum is obtained at a InSb layer thickness of $0.5 \mathrm{ML}$, while the polarization is zero at a InSb thickness of $1 \mathrm{ML}$. This polarization behavior is due to elongated monoatomic islands in the incompletely deposited InSb layer. These kinds of elongated islands have also been observed in InAs submonolayers on GaAs (Refs. 9 and 10) and recently in the case of $\mathrm{InSb}$ grown on GaAs. ${ }^{11}$

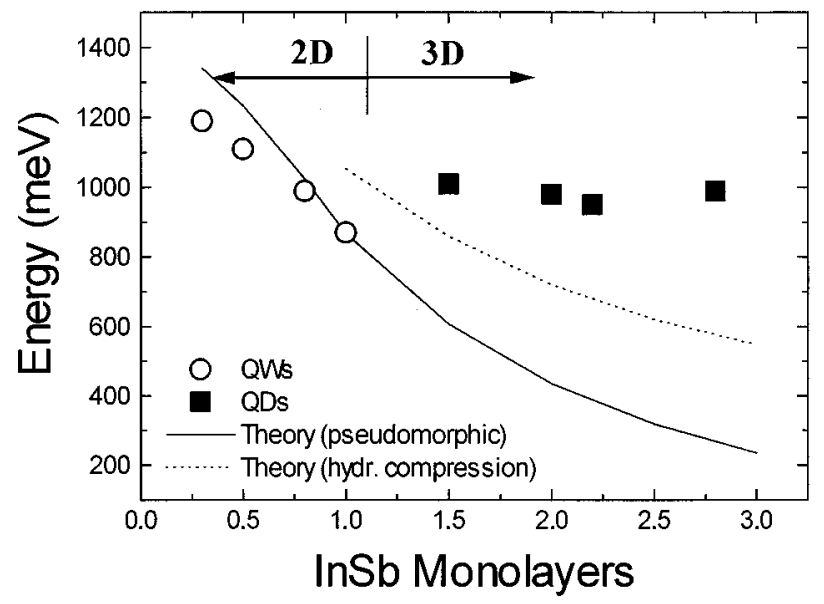

FIG. 3. Experimental emission energies as a function of the Insb coverage for the two-dimensional (2D) grown samples (open circles) and for the QD's samples (3D) (full squares). The lines are the calculated energy positions for the pseudomorphic case (continuous line) and for the situation of Fig. 2(c) (dotted line).

In the dot samples the InSb-related emission cannot be attributed to recombination in the dots. Recently there has been some report about the optical properties of dots in systems with a type-II alignment like $\mathrm{GaSb}, \mathrm{AlSb}$, and $\mathrm{InSb}$ on GaAs. ${ }^{12,13}$ In particular, for GaSb dots on GaAs it has been shown that despite the type-II alignment and the differences between the strain state in the dots and in the QWs the emission related to the dots is located at a lower energy than the emission of the 1-ML QW. We associate the emission observed in the samples with InSb dots with transitions due to the wetting layer. Moreover, in accordance with transmission electron microscopy and Raman measurements ${ }^{14}$ uncapped $\mathrm{InSb}$ dots are completely relaxed, while due to the capping layer there is residual strain present in the dots. In Fig. 2(c) we present the band alignment for this situation assuming that the residual strain is hydrostatic and using the values of this strain measured by Raman. With this band alignment we present in Fig. 3 the theoretical values of the transition for an InSb QW as a function of the QW thickness, this curve serves to estimate the energy position of the transition associated with the dots. According with atomic force micros-

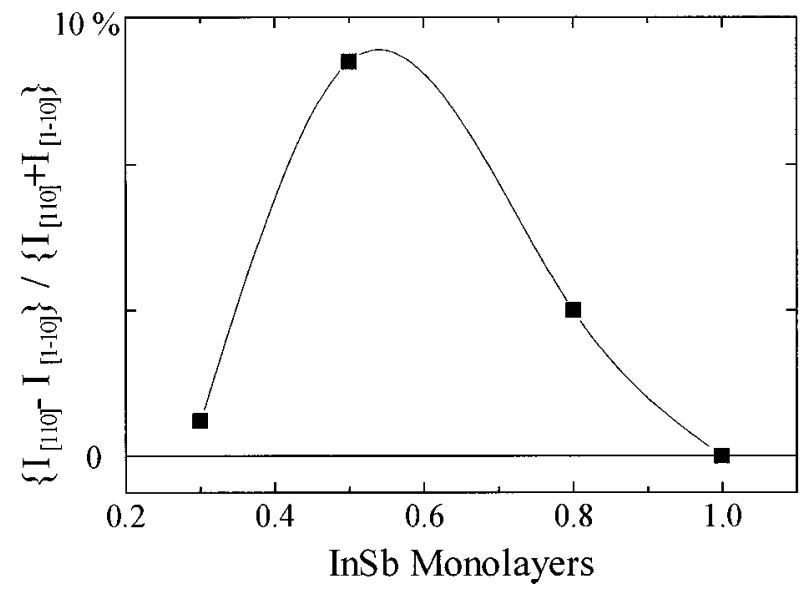

FIG. 4. Linear polarization ratio of the PL signal from the samples with layer thickness from 0.3 to $1 \mathrm{ML}$. 
copy measurement the sizes of the uncapped dots are 90-30 $\mathrm{nm}$ and the height $20-8 \mathrm{~nm},{ }^{3}$ therefore the emission related to the dots is expected to occur at an energy lower than our lower limit of detection $(0.75 \mathrm{eV})$.

To conclude, we have studied the optical properties of $\mathrm{InSb}$ layers in InP with $\mathrm{InSb}$ thickness covering the range between 0.3 and $3 \mathrm{ML}$. In the submonolayer regime the energy position of the InSb related transition decreases as we increase the amount of InSb. The experimental findings sug- gest a type-II alignment for InSb and InP and a VBO similar to the one predicted by the solid-model theory. The PL data indicate that the InSb submonolayer nucleate as twodimensional islands. In the sample with dots emission has been observed which is attributed to the wetting layer.

The authors would like to gratefully acknowledge support by the European Union (HCM, network CT930349) and by CICYT under Project No. MAT95-0966.
${ }^{1}$ E. Michel, G. Singh, S. Slivken, C. Besikci, I. Ferguson, and M. Razeghi, Appl. Phys. Lett. 65, 3338 (1994).

${ }^{2}$ Semiconductors, Physics of Group IV Elements and III-V Compounds, edited by K.-H. Hellwege and O. Madelung, LandoltBörnstein, New Series, Group III, Vol. 17, Pt. A (SpringerVerlag, Berlin, 1982).

${ }^{3}$ T. Utzmeier, P. A. Postigo, J. Tamayo, R. Garcia, and F. Briones, Appl. Phys. Lett. 69, 2674 (1996).

${ }^{4}$ Y. Gonzalez, L. Gonzalez, and F. Briones J. Vac. Sci. Technol. A 13, 73 (1995).

${ }^{5}$ J. Tersoff, in Heterojunction Band Dicontinuities, edited by F. Carpasso and G. Margaritondo (North-Holland, Amsterdam, 1987).

${ }^{6}$ C. G. Van der Walle, Phys. Rev. B 39, 1871 (1989).

${ }^{7}$ D. D. Nolte, W. Walukiewicz, and E. E. Haller, Phys. Rev. Lett. 59, 501 (1987).

${ }^{8}$ F. Hatami, N. N. Ledentsov, M. Grudmann, J. Bohrer, F. Hein- richsdoff, M. Beer, D. Bimberg, S. S. Ruvimov, P. Werner, U. Gösele, J. Heydenreich, U. Richter, S. V. Ivanov, B. Y. Meltser, P. S. Kopev, and Z. I. Alferov, Appl. Phys. Lett. 67, 656 (1995).

${ }^{9}$ T. Ide, A. Yamashita, and T. Mizutani, Phys. Rev. B 46, 1905 (1992).

${ }^{10}$ V. Bressler-Hill, A. Lorke, S. Varma, P. M. Petroff, K. Pond, and W. H. Weinberg, Phys. Rev. B 50, 8479 (1994).

${ }^{11}$ B. R. Bennett, B. V. Shanabrook, and P. M. Thibado, J. Cryst. Growth (to be published).

${ }^{12}$ N. N. Ledentsov, J. Böher, M. Beer, F. Heinrichsdoff, M. Grundmann, D. Bimberg, S. V. Ivanov, B. Y. Meltser, S. V. Shaposhsinikov, I. N. Yassievich, N. N. Faleev, P. S. Kopev, and Z. I. Alferov, Phys. Rev. B 52, 14058 (1995).

${ }^{13}$ E. R. Glasser, B. R. Bennett, B. V. Shanabrook, and R. Magmno, Appl. Phys. Lett. 68, 3614 (1996).

${ }^{14}$ G. Armelles, T. Utzmeier, P. A. Postigo, F. Briones, J. C. Ferrer, F. Peiró, and A. Cornet, J. Appl. Phys. 81, 6339 (1997). 